\title{
Die arthroskopische Behandlung des frühen Gelenkinfektes
}

\author{
Peter Herrmann, Volkmar Heppert
}

\section{Zusammenfassung}

Die Infektion eines Gelenkes ist ein schweres Krankheitsbild, das nur durch frühzeitige Diagnosestellung und umgehende operative Therapie ohne schwerwiegende Folgeschäden geheilt werden kann. Jede akute Gelenkinfektion ist ein Notfall. Kenntnisse der Pathophysiologie und die exakte Klassifikation der Stadien eines Gelenkinfektes sind hierfür unverzichtbar. Gerade in frühen Stadien der Infektion ist die arthroskopische Sanierung anzustreben. In höheren Stadien ist es nur dem in den arthroskopischen Operationstechniken wirklich erfahrenen Operateur möglich, auf minimalinvasivem Wege diesen wichtigen Eingriff rasch, kompetent und notfallmäßig durchzuführen. Die Grenzen, Chancen und die Technik der Arthroskopie eines Gelenkinfektes werden in dieser Arbeit dargestellt.

\section{Arthroscopic Treatment of Early Joint Infection}

Infection of any joint is a very serious complication. Only very early diagnosis and immediate agressive surgical treatment can minimise long-term problems. Any acute joint infection is an emergency case. Knowledge of the underlying pathophysiology and a correct classification determine the mode of surgical therapy. Infections classified up to stage 2 are usually treated by means of arthroscopy. In stage 3 the experience of the surgeon is the most important influencing factor for good long-term results. For the patient sometimes an open revision might be the better choice. Accordingly, this paper deals with the opportunities on the one hand and the limitations on the other hand of arthroscopic treatment for acute joint infection
Die Infektion eines Gelenkes ist ein multifaktorielles Geschehen und stellt nach wie vor große Anforderungen an den behandelnden Arzt. Die Frühdiagnostik einer solchen Infektion ist zusammen mit einem daraus resultierenden aggressiven chirurgischen Vorgehen das entscheidende Kriterium für das spätere Endergebnis des Patienten. Die einwirkende entzündliche Noxe führt erst zeitlich verzögert zu klinisch apparenten Symptomen. Da Infektionen der Gelenke dank den Fortschritten der modernen Chirurgie seltene Komplikationen geworden sind, werden die Frühsymptome oft nicht rechtzeitig korrekt interpretiert. Die klinische Erfahrung zeigt, dass die beginnende Infektion oft zu spät erkannt wird, um diese Folgeschäden minimieren zu können [4]. Dem Faktor „Zeit“ kommt

OP-JOURNAL 2007; 23: 54-59

(c) Georg Thieme Verlag KG Stuttgart · New York somit höchste Priorität zu. Und gerade hier liegt das Problem: Es mangelt häufig an der persönlichen individuellen Erfahrung des Arztes im Umgang mit dieser Komplikation. Unwidersprochen ist aber die Beobachtung, dass nur die sofortige kompetente Behandlung gute Langzeitergebnisse sichert und jede Verzögerung nachteilige Folgen für den Patienten mit sich bringt.

Funktionelle Folgeschäden, persistierende Schmerzen und längere stationäre Behandlungszeiten, in seltenen Einzelfällen sogar der Tod des Patienten sind die Folge zu spät erkannter Infektionen von Gelenken und führen oft $\mathrm{zu}$ erheblichen Haftpflichtforderungen vonseiten der Patienten bzw. deren Angehörigen und zunehmend auch der Kostenträger.

Der Infekt nach Punktion ist extrem selten. Anders berichtet bei Gelenkpunk- tionen von einer Empyeminzidenz von nur 0,034\%。 [2]. Das Risiko steigt erheblich, wenn neben der diagnostischen Punktion zusätzlich eine Injektion in das Gelenk erfolgt, vor allem dann, wenn Kortikoide in Kristallsuspension appliziert werden. Gerade weil hier die Komplikationsrate so niedrig ist, wird nach Punktion so selten rechtzeitig an den Infekt gedacht.

Die kontinuierlich ansteigende Anzahl von Gelenkeingriffen, bedingt durch den fortschreitenden Einsatz minimalinvasiver arthroskopischer Techniken, erhöht rein quantitativ die Anzahl der postoperativen Gelenkinfektionen, ohne dass die relative Komplikationsrate gestiegen wäre. Da sowohl durch die geringere Traumatisierung dieser minimalinvasiven Verfahren im Sinne der Machbarkeit als auch durch den sozialwirtschaftlichen Druck im Sinne der Notwendigkeit die weitaus meisten dieser Gelenkoperationen ambulant durchgeführt werden, ist die postoperative Nachsorge im Vergleich zur stationären Überwachung eingeschräkt. Die Hürde zur direkten operativen Revision ist sicher in der ambulanten Nachsorge im Vergleich höher, wenn eindeutige Infektzeichen fehlen oder nur gering ausgeprägt sind. Im schlimmsten Fall beginnt die Behandlung so erst dann, wenn sich bereits irreversible Knorpelschädigungen manifestiert haben.

\section{Ablauf und Stadieneinteilung des Gelenkinfektes}

Die bakterielle Infektion eines Gelenkes stört zunächst das komplizierte Wechselspiel zwischen der weichen Gelenkinnenhaut, der sog. Synovialmembran, der von ihr produzierten Gelenkflüssigkeit und der Knorpeloberfläche der Gelenkpartner.

Bereits 24 Stunden nach Infektionsbeginn werden lysosomale Enzyme in den Gelenkraum freigesetzt, die der Keimab- 
wehr dienen, aber auch gegen körpereigenes Gewebe aggressiv wirken. Zu diesem frühen Zeitpunkt ist dies aber klinisch noch nicht feststellbar. Erst nach weiteren 2-3 Tagen finden sich beginnende klinische Infektzeichen. Der weitere Verlauf ist dann fulminant. Freigesetzte vermehrt produzierte Glycosamine führen rasch zur Knorpelerweichung und zur Auffaserung der Knorpeloberfläche. Der Zerfall von weißen Blutkörperchen nach Bakterienaufnahme setzt wiederum knorpelschädigende Enzyme frei und die Gelenkzerstörung schreitet kaskadenförmig rasch fort. Die entzündungsbedingten Fibrinbeläge überwuchern letztlich den Knorpel und blockieren zusätzlich die Knorpelernährung, die ja normal zum Großteil über die Gelenkflüssigkeit und nicht über den Blutweg erfolgt [8].

Allein eine Spülung des Gelenkes in der Frühphase mit Entfernung der im Gelenk vorhandenen Leukozyten führt zu einer Verminderung des Gesamtschadens, da die Leukozytenproteinase somit nicht ihre schädlichen Auswirkungen auf den Knorpel ausüben konnte [7] (Abb. 1).

Auch die Gelenkinnenhaut, die Synovia nimmt teil am Prozess der Gelenkdestruktion. Auf das auslösende Agens reagiert die Synovia ab Tag 3 mit einer unspezifischen Hypertrophie unabhängig vom auslösenden Erreger [9]. Bereits nach 11 Tagen kann eine bis zu 5-fache Dickenzunahme der Synovia vorliegen. Zu diesem Zeitpunkt beginnt sie, den Knorpel als sogenannter Pannus zu überwuchern. Die Ernährung der Knorpelzellen wird hierdurch zusätzlich empfindlich gestört, da diese überwiegend durch Perfusion aus der Gelenkflüssigkeit erfolgt. Ab Tag 17 der Gelenkinfektion findet man einen Durchbruch der Gelenkkapsel, die die Gelenkversteifung nach 5 Wochen irreversibel einleitet [9].

Die Entwicklung einer möglichen Arthrosebildung ist deshalb, wie Abb. 2 zeigt, abhängig vom Zeitpunkt und der Art des chirurgischen Sanierungskonzeptes.

\section{Risikofaktoren}

Bei jeder Schwächung der körpereigenen Immunabwehr kann es zur Gelenkinfektion kommen. Auch das Alter hat hierbei einen negativen Einfluss. An weiteren Faktoren finden sich:

- Zustand nach Operation
- Anzahl und Art der eingebrachten Keime

- Einliegendes Implantat oder Prothese Bakterien haben eine ungeheure Affinität zu Metall. Mit einliegendem Implantat benötigt man 1000-mal weniger Bakterien zur Entstehung eines Infektes als ohne Implantat. Dort einmal adhärent, gelingt es dann zusätzlich nur selten ohne Entfernung desselben, diese zu eliminieren.

- Gewebetrauma (Unfall versus iatrogen)

- Fadenmaterial. Insbesondere die nicht resorbieraren Fadenmaterialien (z.B. PDS Kordel etc.), die zur Stabilisierung bzw. Refixation von Sehnenansätzen Verwendung finden, finden wir überzufällig häufig beim Gelenkinfekt als den Infekt unterhaltendes Agens.

- Streuherde, z.B. Zahnwurzelentzündung, Nasennebenhöhlenentzündungen etc., mit Bakterienverschleppung in das Gelenk auf dem Blutweg.

\section{Diagnostik der Gelenkinfektion}

Der oben geschilderte Verlauf des Infektgeschehens verdeutlicht die zeitliche Dringlichkeit der Diagnosestellung dieser Komplikation, da nur die frühzeitige aggressive operative Therapie ein gutes funktionelles Outcome für den Patienten bietet.

Da Infektionen häufig schleichend beginnen, ist oberste Sorgfalt und die Bereitschaft, geringfügige Änderungen im Behandlungsverlauf wahrnehmen zu wollen, unverzichtbar.

\section{Klinik}

Klinische Zeichen der beginnenden Infektion sind in der Frühphase oft nur diskret ausgeprägt. Die klassische Infektzeichen, die aber auch fehlen können, sind:

- Schmerzhafte Bewegungseinschränkung des Gelenkes

- Rötung und Schwellung des betroffenen Gelenkes

- Überwärmung

- Fieber (oft subfebril)

Nach einer Operation bzw. Injektion kann etwa ab dem 3. Tag mit diesen Symptomen in unterschiedlicher Ausprägung gerechnet werden.

Charakteristisch ist nach primär schmerzfreiem Intervall bzw. postoperativer Besserung die plötzliche klinische Befundverschlimmerung

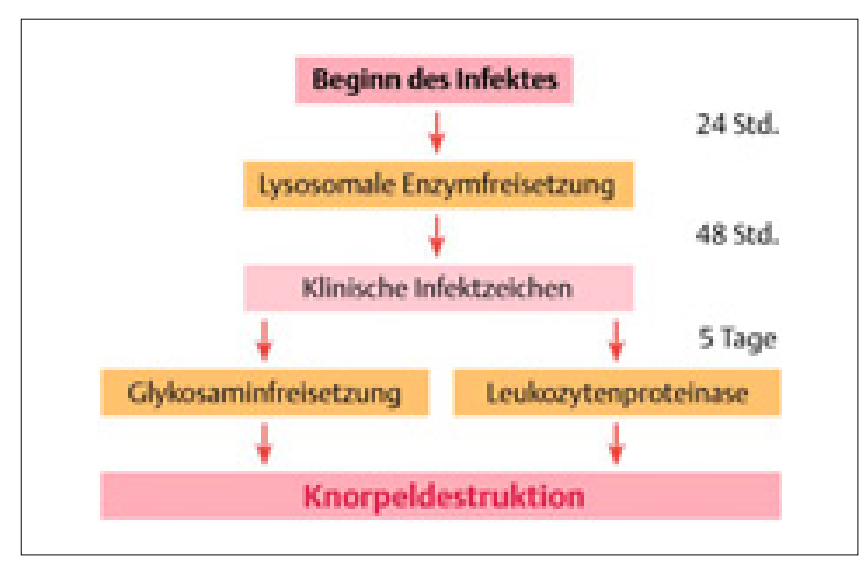

Abb. 1 Infektkaskade im zeitlichen Ablauf [6].

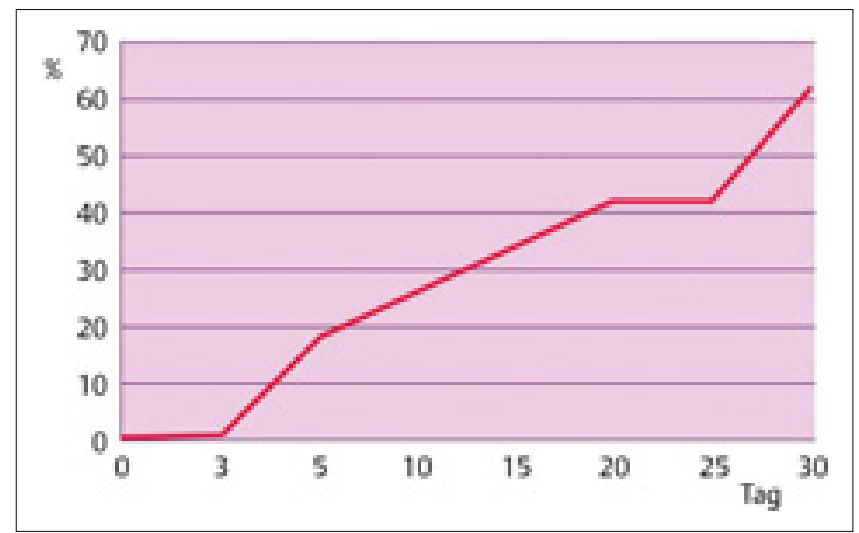

Abb. 2 Entstehung der Arthrose bei Gelenkinfektion im zeitlichen Ablauf [5]. 
Die dann einzuleitende Diagnostik umfasst:

- Leukozytenzahl (unspezifisch)

- Differenzialblutbild

- C-reaktives Protein

- Nativröntgen (Osteolysen?, Materiallockerungen?)

- Gelenkpunktat (unter sterilen Kautelen) bei großen Gelenken. Leukozytenzahl > 30000 Zellen $/ \mathrm{cm}^{3}$ spricht für ein Infektgeschehen

- Bakteriologische Auswertung, Gramfärbung zusätzlich.

Beim akuten Gelenkinfekt darf das mikrobiologische Ergebnis der Punktion nicht abgewartet werden, da hierdurch eine zu große Zeitverzögerung resultiert, deshalb ist die Gramfärbung sinnvoll

- MRT, CT oder Szintigraphie sind ebenso wie spezielle serologische Tests nur bei spezifischer Indikationsstellung, aber nie beim Akutinfekt nach vorausgegangener chirurgischer Intervention erforderlich.

Initiale „blinde“ Antibiotikagaben führen zur zunehmenden Verschleierung der Befunde. Eine definitive mikrobiologische Aussage ist häufig erst nach 8-10 Tagen zu erhalten, zumeist wird zu kurz bebrütet. Hieraus resultieren viele falsch negative Befunde.

Bei eindeutigen klinischen Symptomen der akuten Infektion dürfen deshalb durch die diagnostischen Maßnahmen keine Zeitverzögerungen im Interesse des Patienten resultieren, trotz negativer Kultur kann ein Infekt immer vorliegen.

Keine Punktion unter Antibiotikatherapie zur Diagnose eines Infektes

Keine Punktion mit Lokalanästhesie (kann möglicherweise vorliegende Keime abtöten oder schwächen und ergibt falsch negative Ergebnisse)

Keine definitive Aussage vor 14-tägiger Bebrütung der Abstriche und Kulturen

\section{Klassifikation}

Es gibt in der Literatur kaum einheitliche Klassifizierungen. Die Einteilung nach Gächter findet heute zur Beurteilung großer, der Arthroskopie zugänglicher Gelenke wohl die am weitesten verbreitete Anwendung.

Gächter teilt die Infekte in 4 Stadien ein $[3,10]$ :
- Stadium 1 (Abb. 3)

Trübe Synovia, Rötung der Synovia, mögliche Einblutungen, unauffälliges Röntgenbild

- Stadium 2

Schwere Entzündung mit Fibrinablagerung, Eiter, unauffälliges Röntgenbild

- Stadium 3 (Abb.4)

Syoviaverdickung, Bildung von Gelenkraumunterteilungen, noch unauffälliges Röntgenbild

- Stadium 4 (Abb.5a und b)

Aggressive Infiltration des Pannus, Unterminierung des Knorpels, im Röntgenbild Knochenerweichungen unter dem Knorpel, knöcherne Erosionen und Zystenbildungen

\section{Keimspektrum}

Der am meisten verbreitete Keim ist nach wie vor der Staph. aureus. In den letzten Jahren ist aber eine deutliche Zunahme der koagulasenegativen Staphylokokken nachweisbar. Letztere haben eine besondere Affinität zu Kunststoffen bzw. Metall. Dies muss in die therapeutischen Optionen Eingang finden (z.B. Liegezeit der Saug-Spül-Drainage).

Tab. 1 Keimspektrum von 157 Gelenkinfektionen

\begin{tabular}{lc} 
Staph. aureus & 41 \\
\hline Staph. epidermidis & 26 \\
\hline Enterokokken & 5 \\
\hline Enterobacter & 5 \\
\hline Pseudomonas & 6 \\
\hline Streptokokken & 4 \\
\hline Andere & 2 \\
\hline Steril & 11
\end{tabular}

Auch die Enterokokken sind vermehrt nachweisbar. In etwa $11 \%$ ist ein Keimnachweis trotz manifestem Infekt nicht möglich oder erfolgt, wie oben erwähnt, erst nach 10 bis 14 Tagen, also viel zu spät. Eingang in die Therapie darf dies nicht haben. Die Behandlung muss die gleiche sein (Tab. 1).

\section{Therapie}

Schaut man in die aktuelle Literatur der letzten Jahre, so werden mit bestimmter Regelmäßigkeit immer wiederkehrende Fakten immer wieder kontrovers diskutiert. Einen wirklich einheitlichen Standard der Vorgehensweise im Infektfall kann man nicht erkennen. Grundsätzlicher Diskussionspunkt ist:

\section{Arthroskopische oder offene Revision}

- Diese immer wiederholte Diskussion vernachlässigt die Tatsache, dass nur die wenigsten Gelenke des menschlichen Körpers überhaupt arthroskopierbar sind. Zwar berichtet die Lite-

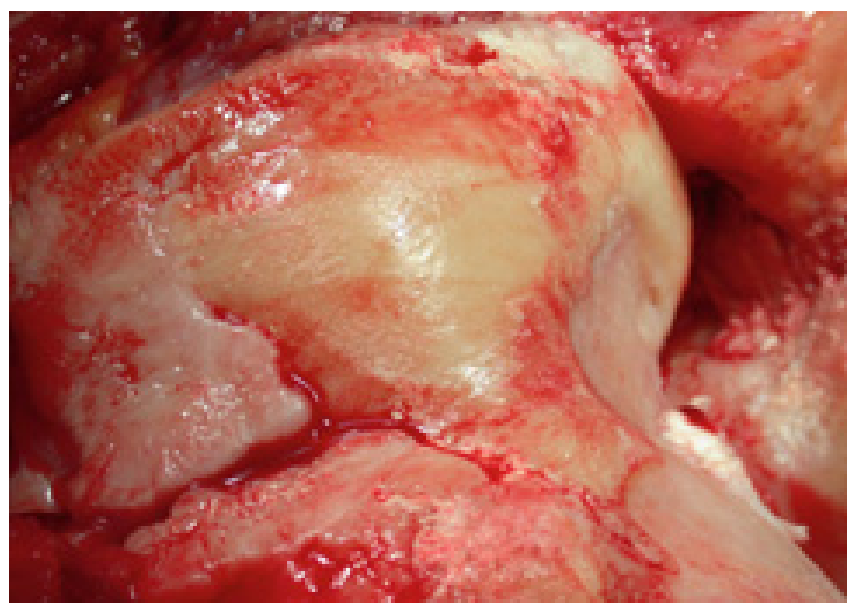

Abb. 4 Synovitis des Kniegelenkes bei Infektstadium 3 nach Gächter mit Überwucherung des Knorpels (hier im Rahmen einer offenen Arthrotomie dargestellt). 

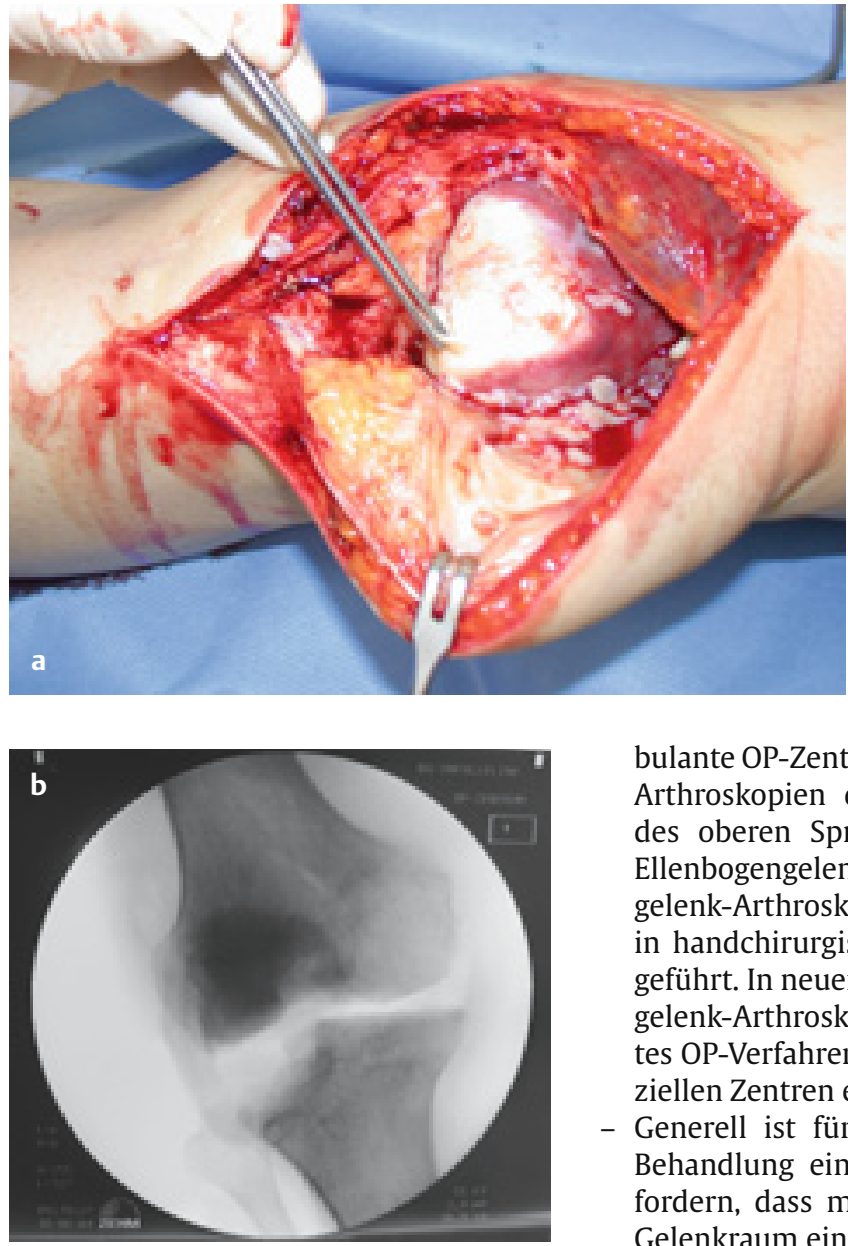

ratur davon, dass sich 95\% aller Infektionen in den großen Gelenken abspielen [8]. Aber die Frage muss erlaubt sein, ob all die Infektionen der kleinen Gelenke in Studien wirklich erfasst werden.

Nehmen wir ein Beispiel: Die Infektion des PIP-Gelenkes des 2. Strahls am Fuß wird durch einfache Hohmann-OP saniert. Bei der scheinbaren Geringfügigkeit des Problems ist davon auszugehen, dass niemand diese Infekte genauso exakt erfasst, wie ein infiziertes Kniegelenk oder Schultergelenk.

Aber dennoch ist auch dieser Infekt unbestritten ein Gelenkinfekt. Bei all diesen kleineren Gelenken muss im Infektfall doch offen revidiert werden. Entzündliche Snovia wird dann mit Messer bzw. scharfem Löffel debridiert und das Gelenk mittels Knopfkanüle mit Ringer-Lactat gespült. Eine sichere Mengenangabe der Spülflüssigkeit gibt es nicht. Hier gilt: „Viel hilft viel“" und dies ist abhängig von der Größe des Gelenkes.

- Das weitaus am häufigsten arthroskopierte Gelenk ist das Kniegelenk. Zunehmend mehr Kliniken und am-
Abb. 5 a Ausgeprägter Infekt des Kniegelenkes Stadium 4, der Gelenkknorpel ist unterminiert und kann mit der Pinzette abgezogen werden.

b Schwerste Gelenkdestruktion Stadium 4 mit Knochendestruktion im Röntgenbild bei Kniegelenkinfektion.

bulante OP-Zentren führen heute auch Arthroskopien des Schultergelenkes, des oberen Sprungelenkes und des Ellenbogengelenkes durch. Die Handgelenk-Arthroskopie wird zunehmend in handchirurgischen Zentren durchgeführt. In neuerer Zeit wird die Hüftgelenk-Arthroskopie als standardisiertes OP-Verfahren etabliert und in speziellen Zentren eingeführt.

- Generell ist für die arthroskopische Behandlung eines Gelenkinfektes zu fordern, dass möglichst der gesamte Gelenkraum einsehbar sein sollte und über den minimalinvasiven Zugang alle Seitenkammern des Gelenkes der Spülung und dem Debridement zugänglich sein müssen. Das Debridement kann schonend und kostengünstig mit dem Saugpunch erfolgen. Durch den Infekt entstandene Septierungen durch Fibrinbeläge müssen arthroskopisch zu entfernen sein, um „tote Winkel“ in der chirurgischen Therapie auszuschließen.

Nur das Gelenk soll beim Infekt arthroskopisch therapiert werden, das man ausreichend sicher und häufig artroskopisch operiert hat und nicht die Gelenke, die man auch schon mal diagnostisch über das Arthroskop „betrachtet“ hat

- Auch bei arthroskopisch extrem spezialisierten Zentren wird der Erfolg im Stadium III nach Gächter nur mit 67\% bei mehrfachen Spülungen mit Debridement dokumentiert [10]. Andere Autoren sehen daher die Indikation zur offenen Revision abhängig von der Dauer der Infektion [8], um auch die tieferen infizierten Synovialschichten $\mathrm{zu}$ erreichen und der Arthroseentwicklung entgegenzuwirken [5].

\section{OP-Technik}

In unserer Klinik werden Patienten mit einer Infektion eines arthroskopierbaren Gelenkes, bei denen kein Implantat nach Fraktur einliegt, primär arthroskopisch revidiert und das Ausmaß der Infektion festgelegt. Es gilt folgendes Schema:

1. Stadium I wird immer arthroskopisch mit Ringer-Lösung gespült.

2. Stadium II wird immer arthroskopisch mit Ringer-Lösung gespült. Auf einen großlumigen Ablauf im oberen Rezessus meist lateral ist zu achten. Der großlumige Ablauf garantiert, dass auch größere Koagel oder Gewebepartikel ausgespült werden können. Der Zulauf erfolgt durchs Arthroskop. Danach erfolgt das arthroskopische Debridement der Synovialmembran, ohne eine absolut vollständige Synovektomie anzustreben. Die Barrierefunktion gegen die Erreger soll erhalten bleiben. Das Debridement kann schonend und kostengünstig mit dem wiederverwendbaren Saugpunch erfolgen.

Nach Debridement wird mit einer Spülspritze von $250 \mathrm{~cm}^{3}$ danach das Gelenk sorgfältig nachgespült (Abb.6). Dies ermöglicht unter hohem manuellen Druck mit Distension der Kapsel das Ausspülen größerer Koagel von Fibrinresten und Zelldetritus.

3. Stadium III wird je nach Erfahrung des zur Verfügung stehenden Chirurgen arthroskopisch oder offen angegangen.

Nicht immer steht im Notfall zu jeder Uhrzeit ein perfekter Arthroskopeur zur Verfügung. Dem Patienten ist dann mit einer offenen Revision oft besser geholfen als mit einer halbherzigen arthroskopischen Revision

In unserem Krankengut sind die Patienten mit Stadium III zumeist vor Verlegung mehrfach arthroskopisch operiert worden, sodass wir dann die offene Synovektomie bevorzugen.

4. Stadium IV muss immer offen revidiert werden, da hier definitionsgemäß eine zusätzliche Knocheninfektion vorliegt.

Am Beispiel des Kniegelenkes sei hier der Ablauf einer Arthroskopie bei Gelenkinfekt dargestellt:

1. Übliche Lagerung mit frei zugänglichem Gelenk entweder mit frei gelagertem Unterschenkel oder aufliegendem Bein auf Lagerungsrolle. 


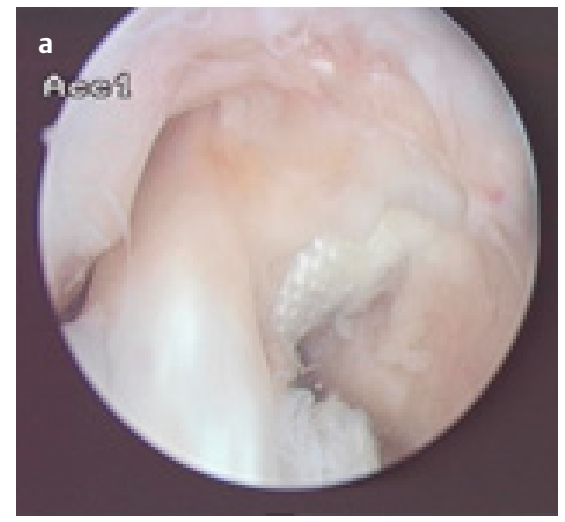

Abb.6a und b a infiziertes Trevira-Kunststoffband als Antiluxationsplastik bei posteriorer Instabilität nach HKB-Ruptur. b gleicher Situs nach arthroskopisch unterstützter Implantatentfernung.

\section{Zulaufkanüle im oberen Recessus und} zuerst Abstrich von der austretenden Gelenkflüssigkeit zur mikrobiologischen Untersuchung. Optikzugang anterolateral.

3. Arthroskopischer Rundgang zur Stadieneinteilung des Infektes.

4. Abtragen von Fibrinbelägen und hypertrophen Synoviazotten, die eine freie Verteilung der Spülflüssigkeit behindern, am schonendsten und günstigsten über einen Arbeitszugang mit dem Saugpunch, der Shaver ist nicht selten zu traumatisierend und zudem aufwendiger und teuer.

5. Fremdmaterialien wie nicht oder sehr langsam resorbierbare Fäden (PDS), Kunstfaserbandersätze (Abb.6a und b) oder Interferenzschrauben mit größerem intraartikulären Anteil müssen als kontaminiert gelten und werden entfernt, sofern es sich nicht um ein ganz frühes Infektstadium handelt. Ein Erhaltungsversuch ist erlaubt. Bandplastiken aus körpereigenem Material werden bei festem Sitz und fehlender Nekrose erhalten.

6. Mechanische Spülung des Gelenkes unter Druck. Dazu Platzierung des Optiktrokars zwischen den Femurkondylen in der sog. Notch vor dem vorderen Kreuzband. Die Spülung erfolgt über den Optiktrokar mit einer 250 ml-Spülspritze. Es sollten mindestens 5 Liter Ringer-Lösung mit manuellem Druck durch das Kniegelenk gespült werden. Voraussetzung ist eine intakte Gelenkkapsel und ein sicherer Ablauf, um eine Verschleppung des Infektes in das Gewebe um das Gelenk auszuschließen (Abb. 7).

Das Arthroskop wird während der Spülung in alle Kompartimente des Gelenkes positioniert, um einen sicheren Spüleffekt überall zu gewährleisten.

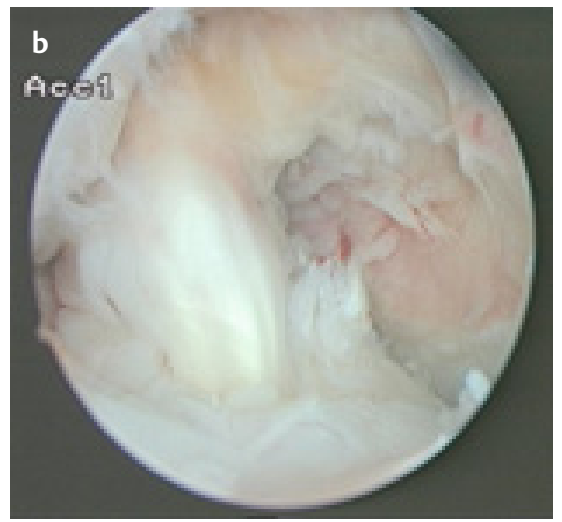

Die 16 Ch. Drainage dient als Ablauf und wird mit dem Schlauchsystem einer Ablaufpumpe verbunden (Abb.9).

Zur Spülung sollte ausschließlich RingerLösung ohne jeden Zusatz verwendet werden. Desinfektionslösungen machen irreversible Knorpelschäden.

Anmerkung:

Die Spül-Saug-Drainage fordert eine exakte Nachsorge mit Bilanzierung von $\mathrm{Zu}$ - und Ablaufmenge und eine ausreichende Entfernung der beiden im Gelenk liegenden Schläuche, um Spülstraßen durch „Kurzschluss“ zu vermeiden. Auch sollte eine gewisse Mobilisation des Gelenkes zur Unterstützung der Flüssigkeitverteilung möglich sein. In unserer Klinik wird die Spül-Saug-Drainage deshalb nach Arthroskopie nur im Kniegelenk angewendet. [1]

Die Spülung erfolgt kontinuierlich über 5 Tage, dann wird an beide Redons ein Vakuumbehälter angeschlossen und die Drainagen werden nacheinander in den beiden folgenden Tagen entfernt.

Wir haben, korrekte technische Durchführung vorausgesetzt, bessere Ergebnisse mit einer Arthroskopie und anschließender SSD am Kniegelenk, als mit wiederholten arthroskopischen Spülungen erfahren.

An den anderen Gelenken wird nach obigem Vorgehen ein antibiotikahaltiger Kollagenschwamm appliziert und die Redondrainage ohne Sog verwendet, um das Antibiotikum nicht abzusaugen.

Eine systemische antibiotische Therapie, testgerecht nach Resistenzlage im Abstrichergebnis wird begleitend durchgeführt über die Dauer von 3 Wochen.

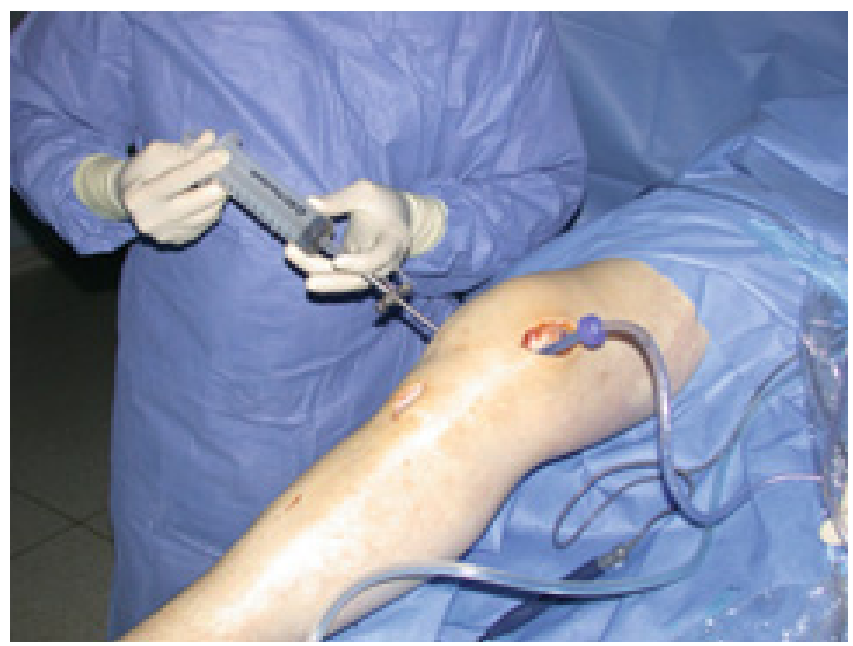

Abb.7 Spülung mit der Spritze über den Optiktrokar unter Druck (der Ablauf liegt hier ausnahmsweise im Knochenkanal eines explantierten HKBErsatzes). 


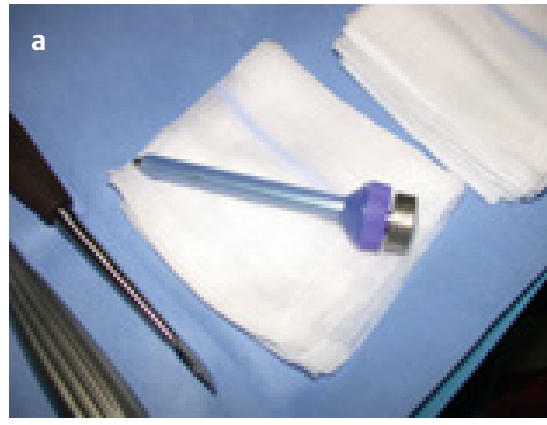

\section{Schlussfolgerung}

Der Infekt eines Gelenkes ist ein schwerwiegendes Krankheitsbild, das sofort nach Diagnosestellung so früh als möglich, also in der Klassifikation als Notfalleingriff in der Bereitschaftszeit, aggresiv chirurgisch angegangen werden muss.

Bei ausreichend sicherer Technik des Operateurs in der operativen Therapie eines Gelenkes auf minmalinvasivem arthroskopischen Wege kann die Therapie aller arthroskopierbaren Gelenke zunächst arthroskopisch erfolgen. Die ausgiebige Spülung und das Abtragen von Fibrinbelägen führt in den Stadien I und II nach Gächter in Kombination mit einer Antibiotikagabe meist zur Ausheilung.

Im Kniegelenk kann mit gutem Erfolg eine Nachbehandlung mit Spül-SaugDrainage erfolgen.

Bei nicht ausreichender Ereichbarkeit aller Gelenkkompartimente bei Stadium III, bei jedem Stadium IV nach Gächter oder wiederkehrenden Infektzeichen nach einmaliger arthroskopischer Therapie empfehlen wir nach wie vor die offene Gelenkrevision und Einlage antibiotikahaltiger Medikamententräger in Form von antibiotikahaltigen KollagenFliesen.

Die persönliche Erfahrung des Behandlers im Umgang mit Gelenkinfektionen ist entscheidend für das Outcome des Patienten.

\section{Literatur}

${ }^{1}$ Albrecht T, Michiels I. Ist die Spül-Saug-Drainage noch zeitgemäß? In: Jerosch (Hrsg.) Infektionen des Bewegungsapparates. Thieme Stuttgart 1995; 9: 45-47

2 Anders G. Gelenkpunktionen und intraartikuläre Injektionen in ambulanten orthopädischen Einrichtungen. Beitr Orthop Traumatol 1984; 31: 419
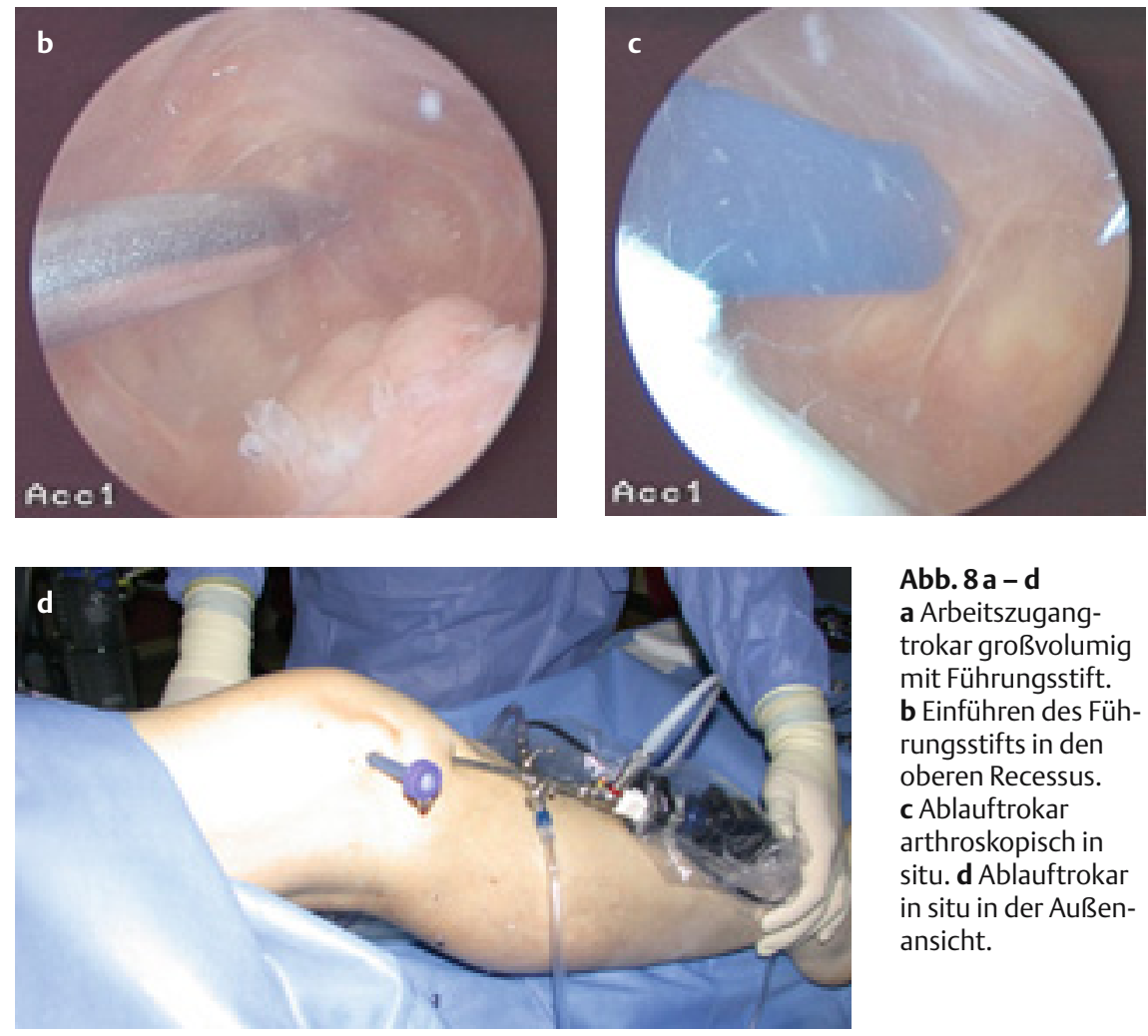

Abb. $8 \mathrm{a}-\mathrm{d}$ a Arbeitszugangtrokar großvolumig mit Führungsstift. b Einführen des Führungsstifts in den oberen Recessus. c Ablauftrokar arthroskopisch in situ. d Ablauftrokar in situ in der Außenansicht.

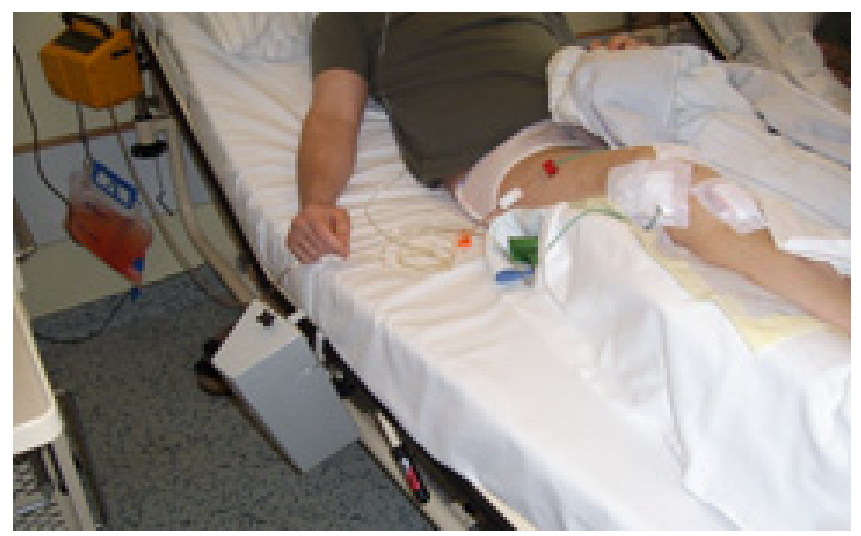

Abb.9 Spül-SaugDrainage des Kniegelenkes mit Ablaufpumpe postoperativ.

3 Gächter A. Der Gelenkinfekt. Inform Arzt 1985; 6: 35-43

${ }^{4}$ Glinz W. Postoperative Gelenkinfektionen Bittere Erfahrungen. Arthroskopie 2001; 14: $1-2$

${ }^{5}$ Härle A, Blauth W, Sönnichsen S, Hepp R. Eine Analyse von 198 Behandlungsergebnissen nach Gelenkinfektionen. Hefte zur Unfallheilkunde 200. 1988; 149-157

${ }^{6}$ Heppert V, Glatzel U, Wagner C, Wentzensen A. Komplikationsmangement bei infizierten Kniegelenken. Trauma Berufskrankh. 2002; 4: 63-71

${ }^{7}$ Holz U., Welte G, Märklin H-M, Weller S. Ergebnisse nach operativer Versorgung von Tibiakopffrakturen. Unfallchirug, 1985; 88: 519-527

8 Jerosch J. Arthroskopische Therapie der bakteriellen Arthritis. In: Jerosch (Hrsg) Infektionen des Bewegungsapparates. Georg Thieme Stuttgart 1995; 9: 38-44

${ }_{9}$ Riegels-Nielsen P, Fridtmodt-Moller N, Jensen JS. Rabbit model of septic arthritis. Acta Scand 1987; 58:14-19
10 Stutz G, Gächter A. Diagnostik und stadiengerechte Therapie von Gelenkinfekten. Arthroskopie 2001 ; 14: 18-22

\section{Dr. med. Peter Herrmann}

Oberarzt Abteilung Septische Chirurgie Dr. med. Volkmar Heppert Chefarzt

Berufsgenossenschaftliche Unfallklinik Ludwigshafen

Ludwig-Guttmann-Straße 13 67071 Ludwigshafen 